\title{
Passive Demodulation of Optical Interferometric Sensors
}

\author{
EUGENE L. GREEN AND PETER G. CABLE
}

\begin{abstract}
Two techniques for passive (no-feedback) demodulation of signals from a remote two-beam interferometric sensor are discussed. Termed "synthetic heterodyne" and "quadrature recombination," both methods are based on forced sinusoidal modulation of phase within the interferometer. If the phase modulation is generated by linearly proportional frequency modulation of the laser light source, then the interferometer, deployed by means of optical fibers, can be a completely passive remote sensor, and an array of identical sensors can be operated from one light source. The former technique, proposed by Cole, Danver, and Bucaro permits standard FM demodulation. The latter technique, proposed by Cable and Green, employs arithmetic operations that are appropriate to digital implementation. Constraints on application of the technique, especially frequency distribution and amplitude of phase noise (out-of-band) versus signal amplitude (in-band), are analyzed and an implementation is proposed that is consistent with contemporary analog-to-digital conversion technology.
\end{abstract}

\section{INTRODUCTION}

$\mathbf{I}^{\mathrm{N}}$ N REMOTE interferometric sensor applications, such as optical hydrophones, weak signal modulations of phase are usually superimposed on much larger fluctuations caused by temperature and pressure variations, and mechanical vibrations. If it can be assumed that signal and fluctuations (or phase noise) are separable in frequency, then it is possible to devise measurement techniques that will permit observation of a stable signal. For example, let $\Delta(t)$ be in-band (high frequency) signal and $\phi(t)$ be out-of-band (low frequency) fluctuation, then it is possible by feedback means to compensate actively for the optical path or phase fluctuations within the interferometer. Thus, if a low frequency feedback signal $X(t)$ is introduced in the carrier phase such that $X(t)=-\phi(t) \pm \pi / 2$ at each instant, then the observed demodulated signal will be proportional to $\sin \Delta(t)$. Such homodyne operation may be achieved either by means of a phase shifter located within the interferometer [1], or by external tuning of the laser frequency to cause a phase change within the interferometer linearly proportional to the frequency change [2]. Alternatively, in principle, a wideband phase-locked loop may be maintained such that $\psi(t)=\phi(t)+$ $\Delta(t)=\pi / 2$ continuously. Thereby an active processor would measure absolute phase independent of multiplicative intensity noise sources [3].

Maintaining phase stability in the presence of very large low frequency fluctuations of path difference between signal and

\footnotetext{
Manuscript received March 26, 1982; revised June 4, 1982. This work was supported in part by the Independent Exploratory Development Program of the Naval Underwater Systems Center and the Naval Research Laboratory FOSS Program.

The authors are with the U.S. Naval Underwater Systems Center, New London, CT 06320.
}

reference beams may require feedback compensation over an excessively large interval compared to the intrinsic frequency or phase modulation capability of the physical control device, whether within the interferometer or external to the interferometer; therefore, rapid phase or frequency flyback may be needed to maintain quadrature. To overcome this constraint, and to provide passive operation (no feedback control) of the interferometer, heterodyne techniques have been proposed. Usually a fixed frequency displacement is imposed upon the light in the reference beam of the interferometer [4]. Recently, Cole et al. [5] and independently, Cable and Green [6] proposed demodulation techniques based upon the imposition of a large sinusoidal modulation of phase within a two-beam interferometer. The motivation of the Cole et al. version was the replacement of a frequency shifter within the interferometer by a more energy efficient phase modulator, which directly causes the sinusoidal phase variation at a fixed frequency. This technique has been termed "synthetic heterodyne" by its originators. The motivation for the Cable-Green development was to directly achieve stable, totally passive operation in the sense of avoiding the need for either an active physical device within the interferometer or for feedback control of the laser frequency. In the Cable-Green approach, termed "quadrature recombination," the laser light source is frequency modulated by a sine wave so as to effect a sinusoidal phase modulation of predetermined amplitude within the two beam interferometer; in this case, a finite known path difference must exist by design between the two beams, so that frequency variations of the light will cause a linearly proportional phase variation. While these methods, synthetic heterodyne and quadrature recombination, differ essentially in the manipulations by which demodulation is performed, both techniques achieve passive operation.

This last point is important, for only passive interferometry is consistent with the preferred optical hydrophone array concept illustrated in Fig. 1. In this sensor array configuration, light from one laser is transmitted by an optical fiber to an array of remote sensors and returned (to the point of origin) by means of a fiber (or multiple parallel fibers) for signal processing and detection. It is apparent that the passive array concept in Fig. 1 is not compatible with signal stabilization techniques that require active elements outboard or with feedback control methods that require a separate laser for each sensor.

As indicated above, the methods by which demodulation is performed differ between synthetic heterodyne and quadrature recombination, and result in different suggested implementation 


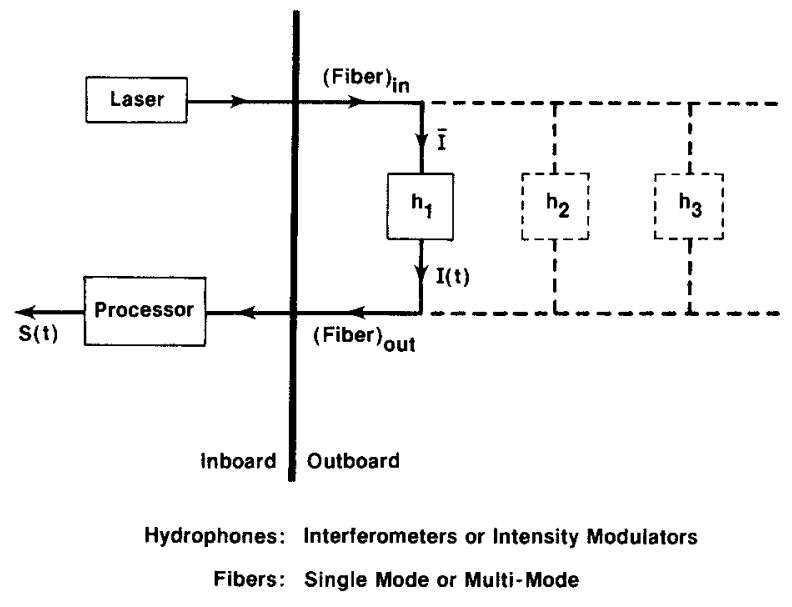

Fig. 1. Optical hydrophone array concept.

approaches. In the Cole-Danver-Bucaro synthetic heterodyne technique, the processor yields at the FM demodulator input a heterodyne signal whose phase modulation is $\psi(t)=\Delta(t)+\phi(t)$ (in-band signal plus out-of-band noise). In practice, the ratio of maximum out-of-band phase fluctuation $\phi(t)$ to in-band signal modulation $\Delta(t)$ may exceed $10^{6}(120 \mathrm{~dB})$. Although applicable FM demodulation is within the capability of contemporary analog electronic technology [7], it is not clear that a compact stable device for use outside of a laboratory environment can be built.

The Cable-Green quadrature recombination technique is based fundamentally upon subtraction of low frequency fluctuation from the total signal, followed by recombination of in-band and out-of-band signal components to yield an output linearly proportional to $\sin \Delta(t)$. The technique is adaptable to analog digital implementation, wherein $\mathrm{A} / \mathrm{D}$ conversion would be done in a moderate dynamic range (consistent with current digital technology) upon four analog signals that represent orthogonal pairs of signal components in the two frequency bands. A limitation on direct application of the technique is the condition that the large out-of-band fluctuations to be subtracted must be confined to very low frequencies, far from the signal band.

It is proposed in this analysis that a division into high frequency and low frequency bands be performed by analog filtering that is optimized to yield four outputs (two for each orthogonal component of the interferometer signal); each of these outputs then may be represented in a dynamic range compatible with practical A/D conversion. The resulting pair of signals for each quadrature component will be recombined by digital addition into one wideband signal. The two orthogonal components thus obtained in digital format will be processed to yield the desired (in-band) signal. The CableGreen technique is very well suited to digital implementation, and the resulting signal output will not be subject to multiplicative (intensity) noise. Other demodulation techniques also may be investigated once a digital representation of the total signal has been obtained.

\section{ANALYSIS}

Consider an optical interferometric sensor that incorporates one input port from which the light splits into two beams, a signal or modulated beam, and a reference beam; the two beams are recombined to exit by one output port. By sinu- soidally modulating the frequency of the light, given a known macropath difference between signal and reference beams, a sinusoidal modulation of the phase difference between the two beams within the interferometer may be imposed. Alternatively, a high frequency sinusoidal phase modulation may be generated directly by a device located in the reference path within the interferometer. In either case, the output of the device, i.e., the intensity of the recombined beams, or the photocurrent from a light detector, will be given by

$$
I(t)=\bar{I}\left\{1+\cos \left[\beta \sin \omega_{r} t+\psi(t)\right]\right\}
$$

where $\omega_{r}$ is the frequency of the imposed phase modulation, $\beta$ is a constant (modulation index), and $\psi(t)$ is the phase modulation attributable to the environment surrounding the interferometer, which includes signal (in-band), and noise (out-of-band) contributions. In the above, high mutual coherence between signal and reference beams and equal intensities have been assumed for convenience.

The signal observed at the output of a band-limited amplifier will be

$$
\begin{aligned}
S(t)= & -2 J_{1}(\beta) \bar{I} \sin \omega_{r} t \sin \psi(t) \\
& +2 J_{2}(\beta) \bar{I} \cos 2 \omega_{r} t \cos \psi(t) .
\end{aligned}
$$

To achieve passive detection, that is, operation with no feedback, we separate the signal $S(t)$ into two orthogonal components

$$
\begin{aligned}
& S_{1}(t)= 2 J_{2}(\beta) \bar{I} \cos 2 \omega_{r} t \cos \psi(t)=2 J_{2}(\beta) \bar{I} \\
& \cdot {\left[\cos \left(2 \omega_{r} t+\psi\right)+\cos \left(2 \omega_{r} t-\psi\right)\right], } \\
& \tilde{S}_{1}(t)=-2 J_{1}(\beta) \bar{I} \sin \omega_{r} t \sin \psi(t)=-2 J_{1}(\beta) \bar{I} \\
& \cdot\left[\cos \left(\omega_{r} t+\psi\right)-\cos \left(\omega_{r} t-\psi\right)\right] .
\end{aligned}
$$

Observe that the filtering operations above require that the frequency $\omega_{r}$ must exceed the width of the sidebands [caused by $\psi(t)]$ extending around $\omega_{r}$ and $2 \omega_{r}$. In practice, $\omega_{r}$ can be made substantially larger than the minimum required.

Cole et al. demodulated $\psi(t)$ by setting $J_{1}(\beta)=J_{2}(\beta) \approx 2.6$. They bring $2 \omega_{r}$ and $\omega_{r}$ to a common carrier frequency $\omega_{o}$ so that the output $S_{o}$ becomes (Fig. 2)

$$
S_{o}(t)=S_{1}(t)+\widetilde{S}_{1}(t)=2 J_{1} \bar{I} \cos \left[\omega_{o} t+\psi(t)\right] .
$$

$S_{o}(t)$ is identical in form to the output of a heterodyne interferometer, within which the reference path includes a device to generate $\left(\omega \pm \omega_{0}\right)$, where $\omega$ is the original optical frequency.

Alternatively, the components $S_{1}$ and $S_{1}$ may be synchronously detected versus their respective subcarriers $2 \omega_{r}$ and $\omega_{r}$. Synchronous detection will cancel phase noise of the reference oscillator (if compensation is made for time delay in propagation to the remote interferometer). Cable and Green, Fig. 3, propose to derive $\sin \Delta(t)$ from the following set of outputs:

$$
\begin{aligned}
\Sigma_{1}(t) & =2 J_{2}(\beta) \bar{I} \cos \psi(t)=2 J_{2}(\beta) \bar{I} \cos [\phi(t)+\Delta(t)] \\
\widetilde{\Sigma}_{1}(t) & =-2 J_{1}(\beta) \bar{I} \sin \psi(t) \\
& =-2 J_{1}(\beta) \bar{I} \sin [\phi(t)+\Delta(t)] \\
\Sigma_{2} & =2 J_{2}(\beta) \bar{I} \cos \phi(t) \\
\tilde{\Sigma}_{2}(t) & =-2 J_{1}(\beta) \bar{I} \sin \phi(t)
\end{aligned}
$$

where $\Sigma_{2}(t)$ and $\widetilde{\Sigma}_{2}(t)$ are obtained by equal low-pass filtering 


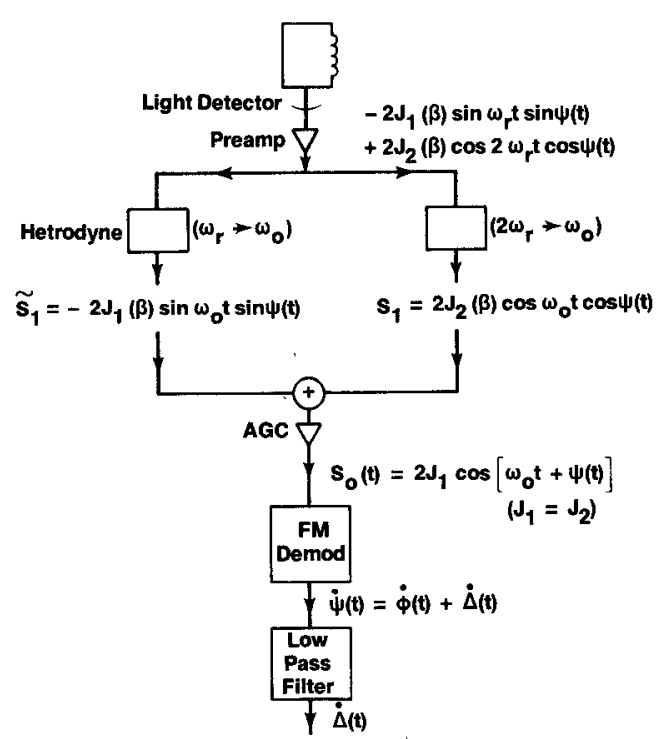

Fig. 2. Analog preprocessing and synthetic heterodyne demodulation.

of $\Sigma_{1}(t)$ and $\tilde{\Sigma}_{1}(t)$, assuming that in-band components of $\cos \phi(t)$ and $\sin \phi(t)$ may be neglected. [This is a restriction on the amplitude and frequency distribution of $\phi(t)$.] Let $\Sigma_{1}(t)$ and $\widetilde{\Sigma}_{1}(t)$ be high-pass filtered prior to processing, or equivalently, let $\Sigma_{2}(t)$ and $\tilde{\Sigma}_{2}(t)$ be subtracted from the total signal to obtain

$$
\begin{aligned}
\delta_{12}(t)= & \Sigma_{1}-\Sigma_{2}=2 J_{2}(\beta) \bar{I} \\
& \cdot[(\cos \Delta-1) \cos \phi-\sin \phi \sin \Delta] \\
\tilde{\delta}_{1.2}(t)= & \tilde{\Sigma}_{1}-\widetilde{\Sigma}_{2}=-2 J_{1}(\beta) \bar{I} \\
& \cdot[(\cos \Delta-1) \sin \phi+\cos \phi \sin \Delta]
\end{aligned}
$$

Then an output linearly proportional to the in-band signal, $\sin \Delta(t)$, may be obtained by either of the following two recombination operations:

$$
O_{1}=\Sigma_{1} \tilde{\Sigma}_{2}-\widetilde{\Sigma}_{1} \Sigma_{2}=4 J_{1}(\beta) J_{2}(\beta) \bar{I} \sin \Delta(t)
$$

or

$$
O_{1}=\delta_{12} \widetilde{\Sigma}_{2}-\widetilde{\delta}_{12} \Sigma_{2}=4 J_{1}(\beta) J_{2}(\beta) \bar{I} \sin \Delta(t) .
$$

In the application of the demodulation techniques, total isolation of the quadratic channels will be impossible. Some effects of imperfect filtering are analyzed in the Appendix. The output of quadrature demodulation is subject to a small amplitude shift in the presence of a weak interaction between channels. Synthetic heterodyne is subject to amplitude and phase error.

If the sidebands caused by low frequency noise are subtracted (filtered) from the original double sideband signal at the carrier level [see (3a) and (3b)], we obtain new signal components $S_{1}^{\prime}(t)$ and $\widetilde{S}_{1}^{\prime}(t)$ centered at $2 \omega_{r}$ and $\omega_{r}$. These components can replace $S_{1}(t)$ and $\tilde{S}_{1}(t)$ in subsequent operations, with the advantage that the dynamic range for $S_{1}^{\prime}$ and $\tilde{S}_{1}^{\prime}$ can be much smaller than for the original signals.

From (5e) and (5f), it is observed that

$$
\begin{aligned}
S_{1}^{\prime}(t)= & 2 J_{2}(\beta) \bar{I}\left\{\operatorname { c o s } \left[2 \omega_{r} t+\cos ^{-1}((\cos \Delta-1) \cos \phi\right.\right. \\
& -\sin \Delta \sin \phi)]+\cos \left[2 \omega_{r} t-\cos ^{-1}((\cos \Delta-1)\right. \\
& \cdot \cos \phi-\sin \Delta \sin \phi)]\}
\end{aligned}
$$

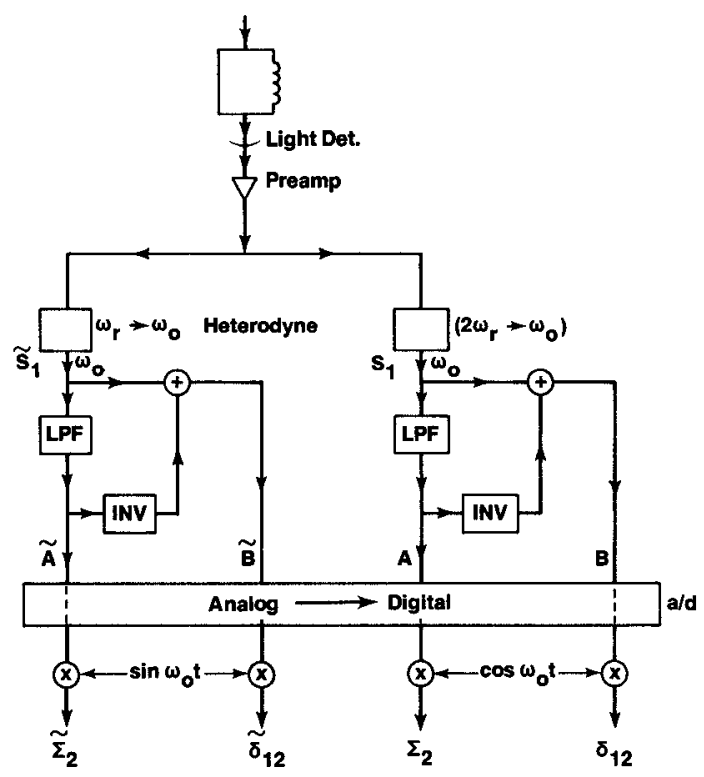

(a)

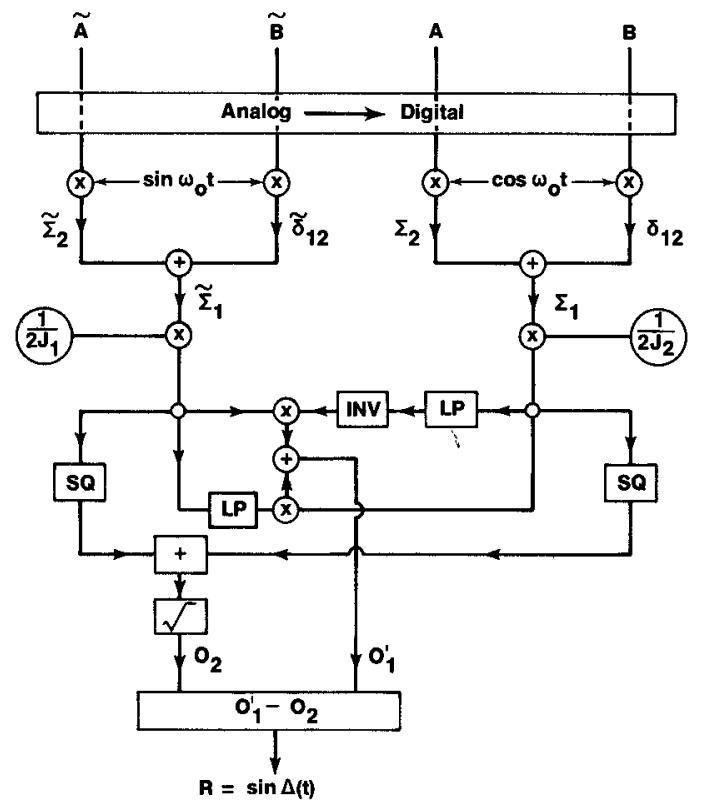

(b)

Fig. 3. (a) Low pass-high pass filtering and A/D conversion. (b) Quadrature recombination.

$$
\begin{aligned}
\tilde{S}_{1}^{\prime}(t)= & 2 J_{1}(\beta) \bar{I}\left\{\operatorname { c o s } \left[\omega_{r} t+\sin ^{-1}((\cos \Delta-1) \sin \phi\right.\right. \\
& +\sin \Delta \cos \phi)]-\cos \omega_{r} t-\sin ^{-1}((\cos \Delta-1) \sin \phi \\
& +\sin \Delta \cos \phi)]\} .
\end{aligned}
$$

It is apparent from $(3 \mathrm{c})$ and $(3 \mathrm{~d})$, however, that direct recombination of $S_{1}^{\prime}(t)$ and $\widetilde{S}_{1}^{\prime}(t)$ to form a heterodyne FM signal (technique of Cole et al.), is not possible unless the in-band signal $\Delta(t)$ is limited to extremely small angles.

The output, (6a) or $(6 \mathrm{~b})$, is a solution in $-\pi / 2 \leqslant \Delta(t) \leqslant \pi / 2$. If $\Delta(t)$ is a small angle, then the output will be very nearly linear. It is important to observe, however, that the signal components $\delta_{12}$ and $\widetilde{\delta}_{12}$ from which $\cos \phi(t)$ and $\sin \phi(t)$ have been filtered, include not only terms proportional to $\sin \Delta(t)$, which approaches $\Delta(t)$ when $O\left(\Delta^{3}\right) \rightarrow O$, but also terms which are $O\left(\Delta^{2}\right)$. The recombination technique, herein described, is the optimum means to obtain an output exactly proportional to $\sin \Delta(t)$, since $O\left(\Delta^{2}\right)$ terms are deleted in the processing. 
It is implicit in the operation described by (6a) and (6b) that $\cos \phi(t)$ and $\sin \phi(t)$ are insignificant in the signal band. The characteristic which distinguishes this technique from the synthetic heterodyne method is that low frequency fluctuations $\phi(t)$ are to be subtracted from the signal initially rather than after detection of the total phase variation $\phi(t)$. It is essential, therefore, that $\cos \phi(t)$ and $\sin \phi(t)$ be separable in frequency from $\sin \Delta(t)$ and $\cos \Delta(t)$, i.e., that the sidebands of $\cos \phi(t)$ and $\sin \phi(t)$ in the signal band be negligible compared to the signal. This restriction on the sidebands of the low frequency fluctuation can be made more explicit. Consider the low-pass filtering operation $h(t)$ applied to $\cos \psi(t)$ and $\sin \psi(t)$ to obtain the outputs defined in (5c) and (5d). If the filtering is not to distort the out-of-band components (to permit successful recombination), we require that

$$
\int h(t-\tau) \cos \phi(\tau) d \tau \sim \cos \phi(t)
$$

with a similar expression for $\sin \phi(t)$.

Let

$$
\int|h(t)|^{2} d t \equiv T
$$

where $1 / T$ defines the lowest frequency of the signal band. For sufficiently slowly varying (low frequency) phase fluctuations, we can write

$$
\phi(\tau) \approx \phi(o)+\dot{\phi}(o) T+\ddot{\phi} \frac{T^{2}}{2}+\cdots .
$$

Representation of $\phi(t)$ by only the first two terms in the expansion yields

$$
\begin{aligned}
\int h(t-\tau) \cos \phi(\tau) d \tau & \approx \int h(t-\tau) \cos [\phi(o)+\dot{\phi}(o) \tau] d \tau \\
& =H[\dot{\phi}(o)] \cos \phi(t) \approx \cos \phi(t)
\end{aligned}
$$

where

$$
H(\omega) \equiv \int h(t) e^{-i \omega t} d t
$$

Thus, distortions at the filter output arise from the third and higher order terms in the expansion (9). Considering only the leading contribution, to insure errors less than $\epsilon \sim 10^{-6} \mathrm{rad}$, we should have

$$
\sqrt{\operatorname{var}(\ddot{\phi})} T^{2}<\epsilon .
$$

This criterion can also be expressed as

$$
\sqrt{\operatorname{var}(\phi)} \cdot{\overline{\omega^{4}}}^{1 / 2} T^{2}<\epsilon
$$

where $\overline{\omega^{4}}$ is the fourth moment of the spectral density of $\phi(t)$.

It is possible that the criterion of negligible (less than $10^{-6}$ rad) sidebands of $\cos \phi(t)$ and $\sin \phi(t)$ will not be satisfied over the full frequency range occupied by $\phi(t)$. It is then preferable in processing to choose the low-pass filter such that the criterion in (13) is satisfied, even though harmonics of $\phi(t)$ in the signal band would then appear as a pseudosignal (or a noise) that is not removable by further processing.

The front-end analog filtering of the double sideband carrier modulation must be compatible with practical digital conversion of the consequent low-band and high-band signals, i.e., a maximum of $8-16$ bits per sample, versus at least 20 bits needed to represent each quadrature component $\cos \psi(t)$ or $\sin \psi(t)$, representing signal plus out-of-band phase noise. It is noted that the sampling rate per channel must be sufficient to recover the maximum baseband signal frequency, but not the carrier frequency $\omega_{0}$ which will be much higher. In the case of acoustic (sonar) signals, the analog filtering can be made equivalent to prewhitening that is performed in amplification of conventional hydrophone signals (with rapid cutoff below the sonar band). The in-band (high pass) signal so obtained will be characterized by an acoustic mean power spectrum equivalent to that from a conventional hydrophone. Therefore, that analog signal certainly can be converted to a digital signal with a fidelity consistent with present sonar array technology. Since small angle signals need not be measured in the out-of-band components, the latter outputs also can be represented essentially without any distortion by A/D conversion in a moderate dynamic range. Although it is possible that the resulting four filtered components will be appropriate for recombination according to (6a) (i.e., without excessive inband signal contribution by harmonics of low frequency phase noise), this assumption is not required. Each pair of high-band and low-band components, now in digital format, will be summed to yield digital equivalents to the total signals $\Sigma_{1}(t)$ and $\tilde{\Sigma}_{1}(t)$. The recombination technique, that has been discussed then can proceed according to (6b) after digital lowpass filtering with low enough cutoff to assure that components thereby obtained, $\Sigma_{2}(t)$ and $\widetilde{\Sigma}_{2}(t)$, will not have measurable sidebands in the acoustic frequency region. Alternative processing techniques also can be investigated once the total signals $\Sigma_{1}(t)$ and $\widetilde{\Sigma}_{1}(t)$ are in digital format.

The digital format for the orthogonal total interferometer signals $\Sigma_{1}(t)$ and $\tilde{\Sigma}(t)$ also will facilitate definitive processing against multiplicative intensity noise via the identity

$$
\left[\frac{\Sigma_{1}(t)}{2 J_{2}(\beta)}\right]^{2}+\left[\frac{\widetilde{\Sigma}_{1}(t)}{2 J_{1}(\beta)}\right]^{2}=\bar{I}
$$

\section{IMPLEMENTATION}

Fig. 2 shows preliminary analog processing, i.e., heterodyning of $2 \omega_{r}$ and $\omega_{r}$ components to a common carrier frequency $\omega_{0}$. Fig. 2 also shows the essential features of the synthetic heterodyne technique. The operations include summation of cosine and sine double sideband modulation components, AGC, demodulation, and low-pass filtering. The analog AGC operates to stabilize carrier amplitude. This stabilization must be effective in the full dynamic region, i.e., to less than one part in $10^{6}$ over a wide frequency range. The normalization principle (14) is applicable to the synthetic heterodyne technique. An AGC operation in this case would be performed immediately following the heterodyne stage of Fig. 2 and, prior to summing, to obtain the synthetic heterodyne signal. 
Fig. 3(a) shows the heterodyning operation to common carrier $\omega_{o}$ followed by identical filtering operations for both cosine and sine components of double sideband modulation. The low-pass filtered outputs $A$ and $\widetilde{A}$ which include lower frequency sidebands (noise), are subtracted (inversion and sum) from the total sidebands to yield outputs $B$ and $\widetilde{B}$ which include higher frequency sidebands (including signal components). The heterodyning to a common carrier frequency $\omega_{0}$ and the method of filtering are chosen to facilitate matching or reciprocity of the low-pass and high-pass operations. Further operations will be performed after A/D conversion. Obviously, an all digital implementation would be preferable immediately following the preamplifier stage. The processing then would be simplified since $\Sigma_{1}$ and $\widetilde{\Sigma}_{1}$ could be synchronously detected versus $2 \omega_{r}$ and $\omega_{r}$ after preamplification and $\Sigma_{2}$ and $\widetilde{\Sigma}_{2}$ obtained by digital filtering. The proposed implementation is believed to be consistent with present technology.

Each of the four outputs $(A, \widetilde{A}, B, \widetilde{B})$ is representable in a moderate dynamic range (approximately 12 bits). The A/D conversion of each signal must be done at a sampling rate appropriate to the highest baseband modulation frequency contained in the output (much lower than the rate needed for the carrier $\left.\omega_{o}\right)$. The resulting four digital signals are multiplied respectively: $(A, B)$ by digital $\cos \omega_{o} t$ and $(\widetilde{A}, \widetilde{B})$ by digital $\sin \omega_{o} t$, to yield $\left(\Sigma_{2}, \delta_{12}\right)$ and $\left(\widetilde{\Sigma}_{2}, \widetilde{\delta}_{12}\right)$.

Fig. 3(b) shows processing based on recombination of orthogonal signal components according to (6a). Digitized total orthogonal signals $\Sigma_{1}$ and $\widetilde{\Sigma}_{1}$ are obtained by summation of low frequency and high frequency components. Assuming that estimates of $J_{1}(\beta)$ and $J_{2}(\beta)$ are known, and that they are nonzero [preferably, $J_{1}(\beta) \approx J_{2}(\beta)$ ], multiplications by $1 / J_{2}(\beta)$ and $1 / J_{1}(\beta)$ are performed on cosine and sine components. The remaining operations are implementation of $(6 a)$ or $(6 b)$ to obtain $O_{1}$, and of (14) to obtain $O_{2}=\bar{I}$. Their ratio is $R=O_{1} / O_{2}=\sin \Delta(t)$. Multiplicative intensity noise in the signal band will be cancelled by the ratio operation. The lowpass filter (digital) is chosen so that sidebands of $\cos \phi(t)$ and $\sin \phi(t)$ do not overlap the signal band.

\section{SUMmARY}

Two proposed basic techniques for passive demodulation of signals from a remote two-beam interferometer have been described. Both are based on sinusoidal modulation of phase within the interferometer. Frequency modulation of the laser light source can generate the required phase modulation within a remote interferometric sensor. Weak (high frequency-in-band) signals are to be detected in the presence of strong (low frequency-out-of-band) phase noise modulations. The synthetic heterodyne technique of Cole et al. leads to an all analog implementation concept. The quadrature recombination technique of Cable and Green is well suited to digital implementation. To facilitate digital conversion of the high dynamic range passive interferometer output, analog prefiltering into low frequency and high frequency subcomponents is proposed which leads to four outputs that may be represented in a reduced dynamic range accessible to contemporary $A / D$ conversion technology. Recombination of these outputs after digital conversion provides the basis for deriving an in-band signal that is stable in the presence of multiplicative intensity noise of laser and fiber optic telemetry elements as well as low frequency phase noise. The proposed quadrature recombination technique is also well suited to operation directly upon unfiltered quadrature components given adequate future means for direct digital conversion of the total analog signals.

\section{APPENDIX}

Let there be imperfect separation of sidebands of $\omega_{o}$ and $2 \omega_{o}$ (harmonic destortion and/or crosstalk) so that the demodulated outputs are

$$
\begin{aligned}
& \Sigma_{1}=2\left[J_{2} \cos \psi-\delta J_{1} \sin \psi\right] \\
& \Sigma_{2}=2\left[J_{2} \cos \phi-\delta J_{1} \sin \phi\right] \\
& \tilde{\Sigma}_{1}=2\left[J_{1} \sin \psi+\rho J_{2} \cos \psi\right] \\
& \tilde{\Sigma}_{2}=2\left[J_{1} \sin \phi+\rho J_{2} \cos \phi\right] .
\end{aligned}
$$

Then, in "quadrature recombination"

$$
\begin{aligned}
\frac{\Sigma_{1} \tilde{\Sigma}_{2}-\Sigma_{2} \widetilde{\Sigma}_{1}}{4 J_{1}, J_{2}} & =[\sin (\psi-\phi)+\delta \rho \sin (\psi-\phi)] \\
& =[1+\delta \rho] \sin \Delta .
\end{aligned}
$$

The consequence of imperfect filtering, defined by (A1a), (A1b), (A1C), and (A1d) is a small amplitude shift in the demodulated output resulting from recombination of the four components.

In "synthetic heterodyne," let $S_{o}$ be the "synthesized" FM signal

$$
S_{0} / 2=\left(\Sigma_{1} \cos \omega_{0} t+\Sigma_{1} \sin \omega_{0} t\right) / 2
$$

where $\Sigma_{1}$ and $\tilde{\Sigma}_{1}$ are given by (A1a) and (A1c).

Let

$$
\begin{aligned}
& J_{1}=k-\mu_{1} \\
& J_{2}=k+\mu_{2}
\end{aligned}
$$

where we assume variation of $J_{1}$ and $J_{2}$ from equality.

Then

$$
\begin{aligned}
S_{o} / 2= & \left(k+\mu_{2}\right) \cos \left(\omega_{o} t+\psi\right)+\delta\left(\mu_{1}-k\right) \sin \left(\omega_{o} t+\psi\right) \\
& +\left(\mu_{1}+\mu_{2}\right) \sin \psi \sin \omega_{o} t \\
& +\left[k(\delta-\rho)-\delta \mu_{1}-\rho \mu_{2}\right] \cos \psi \sin \omega_{o} t .
\end{aligned}
$$

If

$$
J_{1}=J_{2}=k ; \quad \mu_{2}=\mu_{1}=0
$$

then

$$
\begin{aligned}
S_{o} / 2= & k \cos \left(\omega_{o} t+\psi\right)-k \sin \left(\omega_{o} t+\psi\right) \\
& +k(\delta-\rho) \cos \psi \sin \omega_{o} t .
\end{aligned}
$$

The synthetic heterodyne signal is subject to amplitude and phase error as a consequence of crosstalk between channels.

\section{ACKNOWLEDGMENT}

The authors wish to thank J. H. Cole for helpful conversations related to his work. 


\section{REFERENCES}

[1] D. A. Jackson, R. Priest, A. Dandridge, and A. B. Tveten, "Elimination of drift in a single mode optical fiber interferometer using a piezoelectrically stretched coiled fiber," Appl. Opt., vol. 17, pp. 2926-2929, 1980.

[2] P. Shajenko and E. L. Green, "Signal stabilization of optical interferometric hydrophones by tuning the light source," Appl. Opt., vol. 19, pp. 1895-1897, 1980.

[3] A. Olsson, C. L. Tang, and E. L. Green, "Active stabilization of a Michelson interferometer by an electrooptically tuned laser," Appl: Opt., vol. 19, pp. 1897-1899, 1980.

[4] M. A. Nokes, B. C. Hill, and A. E. Barelli, Rev. Sci. Instr., vol. 49, pp. 722-728, 1978.

[5] J. H. Cole, B. A. Danver, and J. A. Bucaro, "Synthetic-heterodyne interferometric demodulation," IEEE J. Quantum Electron., vol. QE-18, pp. 694-697, Apr. 1982.

[6] P. G. Cable and E. L. Green, "Signal processor for optical interferometric hydrophone," Invention disclosure, Naval case 65786.

[7] W. H. Glenn, "Investigation of techniques for the detection of small phase shifts in optical fibers," United Technol. Res. Center, East Hartford, CT, June 1979, Rep. R79-924576-1 (prepared for NRL under contract N00173-79-C-0421.)

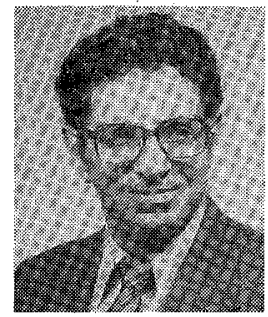

Eugene L. Green received the B.S. and M.S. degrees from Carnegie-Mellon University, Pittsburgh, PA, and the Ph.D. degree from Temple University, Philadelphia, PA, all in physics.

Since 1965, he has been a Research PhysicistConsultant with the Naval Underwater Systems Center, New London Laboratory, New London, CT. Previously, he was with the U.S. Army, Frankford Arsenal, Philadelphia, PA. He has been working for several years in the area of optical sensors and fibers. His other interests have included optical processing, holographic interferometry, and acoustic signal processing for detection and classification. Earlier research actively included optical image analysis and a study of optical properties of alloys in relation to band structure. He conducted the basic laboratory laser experiments that resulted in the $Q$-switch device employed in the first single pulse laser rangefinder. He has publications and patents in these areas.

Dr. Green is a member of the Optical Society of America.

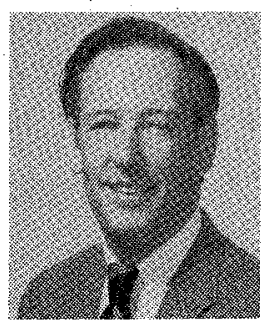

Peter G. Cable received the B.A. degree from Haverford College, Haverford, PA, the M.A. degree from Columbia University, New York, NY, and the Ph.D. degree from the University of Maryland, College Park, all in physics, in 1958,1960 , and 1966 , respectively.

While at the University of Maryland, he was a NASA Predoctoral Fellow. Since 1966 he has been with the Naval Underwater Systems Center, New London, CT. During the academic year 1969-1970, he held a faculty appointment at the Institute for Fluid Dynamics and Applied Mathematics, University of Maryland. From 1973 to 1976 , while at the Naval Underwater Systems Center, he was Head of the Applied Physics Group, Office of the Associate Technical Director for Science and Technology, and from 1976 to 1980 he was Head of the Advanced Systems Technology Division, Special Projects Department. Currently, he is on the staff of the Surface Ship Sonar Department Head. His research interests include the areas of stochastic effects in underwater sound transmission, statistical communication and detection theory, and the modeling of acoustic self and radiated noise.

\title{
Polarization Fading in Fiber Interferometric Sensors
}

\author{
DAVID W. STOWE, DOUGLAS R. MOORE, AND RICHARD G. PRIEST
}

\begin{abstract}
Mach-Zehnder interferometer sensors fabricated with conventional nonpolarization-preserving fibers are subject to polarization fading caused by temperature variations and minor positional changes in the sensor. For such sensors, we calculate the probability of a given decrease in sensitivity and in signal-to-noise ratio due to fading assuming the polarization of the light in the signal and reference legs is uncorrelated and drifts randomly. The resultant reduction of the signalto-noise ratio may exceed $10 \mathrm{~dB} 10$ percent of the time and exceed $20 \mathrm{~dB}$ more than 2 percent of the time.
\end{abstract}

Manuscript received April 1, 1982; revised June 18, 1982.

D. W. Stowe and D. R. Moore are with Gould Laboratories, Rolling Meadows, IL 60008.

R. G. Priest is with the Optical Sciences Division, Naval Research Laboratory, Washington DC 20375.

\section{INTRODU CTION}

$\mathrm{F}$ IBER optic Mach-Zehnder interferometers have been investigated for sensing a variety of environmental parameters such as acoustic pressure [1]-[4], acceleration [5], temperature [6], and magnetic field [7]. In these sensors, the environmental parameter of interest alters the phase of the light in the signal fiber relative to the phase of the light in the reference fiber (Fig. 1), causing a shift in the resultant interference pattern.

Although the all-fiber interferometer is very sensitive, its performance may be degraded by polarization fading which occurs when the two interfering beams approach quasiorthogonal polarization states. For interferometric sensors 